EUROPEAN ORGANIZATION FOR NUCLEAR RESEARCH

CERN-PPE/94-25

11 January 1994

\title{
A Fast Signal Adder for Applications with Calorimeters
}

\author{
J.M. Seixas ${ }^{* \dagger}$, L.P. Caloba ${ }^{\dagger}$ and M.N. Souza ${ }^{\dagger}$
}

\begin{abstract}
An active adder circuit is presented. It is designed to handle fast signals (risetime $\sim 1 \mathrm{~ns}$ ) that have to be linearly combined while good DC stability is to be maintained. This is useful in many fields of application, including calorimeters where the circuit has successfully been used. Experimental results of the adder performance and applications are shown.
\end{abstract}

(Submitted to Nuclear Instruments and Methods)

${ }^{*}$ CERN, Geneva, Switzerland

${ }^{\dagger}$ COPPE/EE/UFRJ, Rio de Janeiro, Brazil 


\section{Introduction}

An active adder that can linearly combine fast signals has many applications. In particular, in High-Energy Physics, such a need comes easily in calorimeters. These detectors are mainly used to measure the energy of the incoming particles; although other useful information can be extracted from them, including particle identification and position resolution [1].

In order to achieve good granularity for their measurements, calorimeters are usually made of thousands of modules (or cells). The combination of a restricted number of these cells is important to help achieving local decision for the triggering system, in which events in a given experiment are selected according to the physics one is searching for. The trigger system in many such experiments is composed of different levels of decision. In the operation of the first level trigger, a region of interest is normally identified and the fast signals coming from the calorimeter in this region are combined so that a fast decision can be reached for each event.

At the moment, detectors are being developed to face the harsh environment represented by the new high-luminosity multi-TeV collider experiments, like the LHC. Event rates of $10^{8-9} / \mathrm{sec}$ characterize these experiments as a very demanding environment for detectors, in which calorimeters play an important role [2].

At CERN, a fiber calorimeter has been developed as a fast and accurate device adapted to the environment of these new collider machines [3]. It's based on a large number of scintillating plastic fibers embedded in a lead matrix. This calorimeter exhibits very fast signal capabilities [4, 5]. Figure 1 shows the signal coming from adding the seven innermost modules of the calorimeter (with respect to the beam interaction point) for a particle beam of $150 \mathrm{GeV}$ electrons. Full width half maximum (FWHM) of 5 ns and rise times of $\sim 3$ ns have been measured on such signals.

In order to linearly mix these fast signals from different cells of the calorimeter, the adder we describe here was used. The main features required from this adder were:

1. Capability in handling fast signals, which can be translated in a step response with a rise-time $\sim 1 \mathrm{~ns}$ and small overshoot.

2. Low-noise performance to allow covering low-energy measurements.

3. Low crosstalk between the input lines. 
4. Good linearity over the whole range of energy.

5. Good performance over the low-frequency range to avoid the introduction of additional tails on the input signals.

6. Low input and output offsets.

7. Full $50 \Omega$ matching for the input and output lines to avoid signal reflections.

In this paper, the adder is described. In Section 2 the circuit topology used in the design is shown. Section 3 covers the description of the measurements performed and Section 4 discusses the applications in which the good performance of the adder was explored. Conclusions are addressed in Section 5.

\section{Circuit Description}

Figure 2 shows the schematics of the active adder we have designed. It supports up to six input signals and provides two output lines. Both input and output lines are $50 \Omega$ matched.

Each input is buffered to assure that the incoming signals can only travel in the forward direction. The output stage combines the signals coming from the six inputs and provides the desired unitary gain (adjustable) to each output line. Wide bandwidth is carried out through the whole design to assure high frequency response (risetime $\sim 1 \mathrm{~ns}$ ).

A compensation for the input and output offsets is provided, besides a compensation for the low-frequency response (tilt). Only one potentiometer is provided for each offset compensation and this has proved to be enough from the measurements performed on the adders (see next section). These compensations are crucial for the applications we will show next, as the offsets would not allow different adders to be combined and the deterioration of the low-frequency response would have brought undesired tails to the fast signals acquired from the calorimeter.

\section{Performance of the Adder}

Thirteen adders were built for the purpose of data acquisition with the calorimeter. On these adders, different measurements were performed to 
evaluate the design. The results described next are average values (or worst case values, when stated) for the total set. The measurements were performed using a digital storage oscilloscope (DSO) ${ }^{1}$ and a charge ADC ${ }^{2}$. Pulses were injected using an arbitrary waveform generator ${ }^{3}$.

\subsection{Linearity and Input Range}

The desired unitary gain for each input signal was adjusted and was accurate (for each input) up to the $5 \%$ tolerance figure of the resistors used. The linear range of the adders was found to be $+100 \mathrm{mv} \rightarrow-1.4 \mathrm{~V}$ into a $50 \Omega$ load (both adder's output connected). The linearity was measured using the charge ADC, with negative step pulses from the generator being injected in the adders and having their amplitude varied while the gate width of the ADC was kept constant (400 ns). All inputs of the adders were excited through power dividers, which allowed to obtain half power signals while keeping the line matched in $50 \Omega$. Around 3000 events were accumulated and the mean value of the distribution found for each input amplitude was used.

The result obtained can be seen in Figure 3. Here the average values for the input and adder's output signals are plotted. Some instability on the amplitude of the signal coming from the generator and the presence of noise on the small amplitude input signals contributed to mask the final result of the adder's linearity performance. However, performing the best linear fit to the experimental values and measuring the deviation of the measured curve from the fit, we found $+0.8 \%,-0.6 \%$ over the linear range, without unfolding any of these instrumental effects.

\subsection{Frequency Performance and Offsets}

The fast signal response feature of the adder can be seen in Figure 4. The response to a fast block pulse reveals a rise-time of $918 \mathrm{ps}$ when the measured value for the rise-time of the input pulse $(882 \mathrm{ps}$, coming from the convolution of the scope's amplifier and generator responses) is unfolded. Similar results we get for the step response of the adder (unfolded rise-time $\sim 1.1 \mathrm{~ns}$ ). Due to overshoots observed in all input signals we had available, the overshoot due to the adders could only be estimated to be less than $5 \%$.

\footnotetext{
${ }^{1}$ Tektronix DSA $602 \mathrm{~A}$ with $400 \mathrm{MHz}$ bandwidth amplifier, 11A32

${ }^{2}$ LeCroy 2280 System with $2282 \mathrm{E}$ modules

${ }^{3}$ Analogic 2045
} 
The tilt of the adder's output was adjusted to be better than $1 \mathrm{mV}$ for all inputs. Therefore, tail effects introduced by the adder can be considered insignificant. Also, input and output offsets were adjusted to be less than 1 $\mathrm{mV}$ and $0.1 \mathrm{mV}$, respectively, for all six inputs and the two output lines.

\subsection{Noise and Crosstalk}

The noise performance was measured using different methods. Firstly, a measurement was performed using the DSO. The adder's inputs were loaded with standard $50 \Omega$ loads and more than 2000 events were accumulated with 10240 points/waveform selected in the DSO. The measured value of 190 $\mu V_{R M S}$ has to be further unfolded from the noise measured ( $\left.124 \mu V_{R M S}\right)$ for the input amplifier of the DSO. This value for the noise figure of the adder was in good agreement with the measurement performed with an analog scope ${ }^{4}$ using a tangential method [6].

A third method for noise evaluation was to use the same charge ADC referred to before. This measurement is interesting as this ADC is being used in the acquisition chain for the calorimeter signals. Therefore, we can measure the noise in terms of ADC counts (and hence in energy) and see how suitable these adders will be for combining calorimeter signals over a wide range of energy. Figure 5 shows the result, when all the inputs of the adders had standard $50 \Omega$ loads and $\sim 10000$ events were accumulated. For this measurement, an active splitter with two output lines (gain 1 and $\sim$ 10) was used to avoid loosing accuracy due to ADC's pedestal values. The calorimeter signals are also acquired by two channels using the same active splitters configuration during beam tests (see Figure 6). The plot shown in Figure 5 refers to the amplified output line of the splitter and the ADC has a gain of 4 counts $/ \mathrm{pC}$. The noise coming from the adder has a mean value of $0.095 \mathrm{pC}$, which would represent $\sim 25 \mathrm{MeV}$ for the calorimeter acquisition purpose $(4 \mathrm{pC} / \mathrm{GeV}$ is being used in the calibration of the detector). It should be noted that no attempt to unfold the noise figure coming from the ADC and the amplified channel of the splitter has been realized.

The crosstalk between the input lines of the adder was measured to be less than $61 \mathrm{~dB}$, for all inputs.

\footnotetext{
${ }^{4}$ Tektronix $2565 \mathrm{~A}$
} 


\section{Applications}

We used the adder in the fiber calorimeter's acquisition chain. The main application in our case was to combine pulses coming from different cells of the calorimeter to build the signal to be sent to an electron/pion discriminating system. Such a system [7] was designed to operate in the online analysis (first level trigger) of the calorimeter in a LHC like experiment, recognizing electrons based on the calorimeter signal structure. The signal to be discriminated was selected to be the one coming from adding the seven innermost towers of the calorimeter (see Figure 1) with respect to the interacting point. When the detector prototype used was a monolithic projective calorimeter [8], each tower had two separate readouts, defining electromagnetic and hadronic sections. Therefore, to obtain the discriminating signal based on the seven innermost towers, we had to add 14 signals. Some other studies for shower development profiles required the acquisition of more towers, which used the total 13 adder units and implied a six input configuration to be the optimal design for the adder.

Figure 6 shows the acquisition chain used for this projective calorimeter prototype. The line is matched through cables on both branches, keeping a $50 \Omega$ matching all along the blocks of the chain. The signal from the photomultiplier (PM) base goes to a charge ADC (through an active splitter to circumvent dynamic range limitations on this branch) and a combination of such signals (through our adder) is sent to a DSO using a fast cable to avoid long tail effects. The adder provides a split output (same gain), which allows monitoring the signals being acquired on ADCs 3 and 4. A pyramidal connection of the adders acquired the discriminating signal, avoiding effects coming from possible different delays of each active adder unit.

The performance of this discriminating system using the sum of seven signal reaches $98 \%$ efficiency in detecting electrons while achieving a rejection factor for pions of 1000 , after some signal processing on the original signal [7]. Here, we need the main features of the adder: its fast $50 \Omega$ matched response and the absence of tails coming from its high quality low-frequency performance and low offsets.

The low-noise characteristic of the adder can also be explored when cells having lower-energy deposition have to be combined. In this case, combining such cells by software (that is, off-line) is hard, as the ADC's pedestal imposes severe restrictions on the low-signal acquisition mode (even when the amplified channel is used). Therefore, one would like to combine these cells 
by hardware using an active adder. However, this adder has to exhibit a good noise figure in order to avoid deterioration of the calorimeter resolution.

As in the acquisition chain (Figure 6; ADC 3,4) we have access to the energy coming from the hardware sum of the cells, we could test whether the adders would deteriorate the electromagnetic resolution of the calorimeter, using the sum of seven signal. Looking through an energy range going from $20 \mathrm{GeV}$ to $150 \mathrm{GeV}$, there was no noticeable difference when we compare the energy resolution measured by the charge ADC combining by software the required 14 towers with the same measurement coming from the hardware combination of towers using the proposed adder.

The adder is also useful when one analyses the calorimeter performance for short acquisition time systems, where ring sum signals (based on the concept of the shower development on concentric cylinders) were often taken as the main feature (for shower profiles studies, etc) [5]. The ring sums in this case have to be performed with adders with a fast signal response, as signals must not suffer from long tail effects (due to the short acquisition time). Also, for the outermost rings, as the tail energy is quite small, low-noise figures for the active adder are required.

\section{Conclusions}

An active adder design was described. It exhibits very good features in terms of noise, linearity and crosstalk. Also, it has capabilities to deal with very fast signals. It has been used in calorimetry, both in particle discrimination and low-energy signal acquisition.

\section{Acknowledgements}

We are thankful for the support that has been provided to this work from Conselho Nacional de Desenvolvimento Científico e Tecnológico (Brasília, Brazil), World Lab ( Lausanne, Switzerland) and CERN (Geneva, Switzerland). We would like to thank the SPACAL group and the RD1 Collaboration, within which the circuits and the applications described here have been developed. In particular, we thank M. Gaspar, A. Braga and B. Tomé for their help in building the circuits and performing some of the tests. 


\section{References}

[1] Wigmans, R. Rev. Nucl. Part. Sci. 41 (1991) 133

[2] Proceedings of the II and III International Conference on Calorimetry in High Energy Physics. Capri, 1991/ Corpus Christi, 1992. Published by World Scientific.

[3] Acosta, D. et al. Nucl. Instr. and Meth. A(308) 4811991.

[4] Acosta. D. et al. Nucl. Instr. and Meth. A(302) 361991.

[5] Acosta, D. et al. Nucl. Instr. and Meth. A(314) 4311992.

[6] Franklin, G. and Hatley T. Electronic Design 24, November 22, 1973.

[7] Fast Front-End Electronics for Electron/Pion Separation in a Lead/Scintillating Fiber Calorimeter. Seixas, J.M. Proceedings of the II Int. Conf. on Calorimetry in High-Energy Physics, Capri, Italy, 1991. Published by World Scientific, 1992, p 445.

[8] Badier, J. et al. Nucl. Instr. and Meth. A(337) 3261994.

\section{Figure Captions}

1. The calorimeter signal coming from adding its seven innermost cells for $150 \mathrm{GeV}$ electron beam. The sampling rate is $1 \mathrm{GSa} / \mathrm{s}$.

2. The circuit diagram of the active adder. The input circuit for each of the six inputs (a), the output section (b) and the low-frequency/offset compensation networks (c) are shown. The NPN transistors are BFR90, the PNP one is BFQ32 and the operational amplifier is a 741 type. Resistors are in ohms.

3. The linearity of the adders. Values are in $\mathrm{pC}$ and the $\mathrm{ADC}$ has a gain of 4 counts/pC.

4. The adder response (bottom) to a fast block pulse (top).

5. Noise figure for the adder using a charge ADC.

6. Acquisition chain for the calorimeter signals. 